\title{
ON THE INNER AUTOMORPHISMS OF FINITE TRANSFORMATION SEMIGROUPS
}

\author{
by INESSA LEVI
}

(Received 20th December 1993)

\begin{abstract}
If the group of inner automorphisms of a semigroup $S$ of transformations of a finite $n$-element set contains an isomorphic copy of the alternating group Alt $_{n}$, then $S$ is an $S_{n}$-normal semigroup and all the automorphisms of $S$ are inner.
\end{abstract}

1991 Mathematics subject classification: 20M20.

\section{Introduction}

Given a semigroup $S$ of transformations of a set $X_{n}=\{1,2, \ldots, n\}$, denote by $G_{s}$ the subgroup of the symmetric group $S_{n}$ of all the permutations $h$ of $X_{n}$ satisfying $h S h^{-1} \subseteq S$. Therefore for each $h \in G_{S}$, the mapping $\phi_{h}: S \rightarrow S$ defined by $\phi_{h}(\alpha)=h \alpha h^{-1}$, for $\alpha \in S$, is an automorphism of $S$. Such an automorphism of $S$ is termed inner [5] and the set of all inner automorphisms of $S, \operatorname{Inn} S=\left\{\phi_{h}: h \in G_{s}\right\}$, forms a subgroup of the group Aut $S$ of all automorphisms of $S$.

Observe that if $S=T_{n}$, the semigroup of all total transformations of $X$, then $G_{S}=S_{n}$. A subsemigroup $S$ of $T_{n}$ is said to be $S_{n}$-normal if $G_{S}=S_{n}$. In this case all the automorphisms of $S$ are inner, and Aut $S=\operatorname{Inn} S \cong S_{n}[6]$.

The main result of this paper asserts that if $G_{S}$ contains the alternating group Alt $_{n}$ then $G_{S}=S_{n}$, so that $S$ is an $S_{n}$-normal semigroup, and Aut $S=\operatorname{Inn} S \approx S_{n}$. Therefore, there is no $S \subseteq T_{n}$ such that $G_{S}=\mathrm{Alt}_{n}$.

We generally use letters $h, p, g$ to denote permutations of $X_{n}$, and $\alpha, \beta, \gamma, \delta$ to denote non-permutations in $T_{n}$. In the following series of results we prove the theorem stated below.

Theorem. Let $S$ be a subsemigroup of $T_{n}, n \geq 3$. If the group Inn $S$ contains a subgroup $G$ isomorphic to Alt $_{n}$ then Aut $S=\operatorname{Inn} S \cong S_{n}$, and $S$ is an $S_{n}$-normal semigroup.

Given $\alpha \in T_{n}$ and a subgroup $G$ of $S_{n}$, let $\langle\alpha: G\rangle=\left\langle\left\{h \alpha h^{-1}: h \in G\right\}\right\rangle$ be the subsemigroup of $T_{n}$ generated by all the conjugates of $\alpha$ by the elements of $G$. Observe that if $\beta \in\langle\alpha: G\rangle$, then $\beta=h_{1} \alpha h_{1}^{-1} h_{2} \alpha h_{2}^{-1} \ldots h_{k} \alpha h_{k}^{-1}$ for some $h_{1}, h_{2}, \ldots, h_{k} \in G$, and so for any $h \in G, h \beta h^{-1}=h h_{1} \alpha h_{1}^{-1} h^{-1} h h_{2} \alpha h_{2}^{-1} h^{-1} \ldots h h_{k} \alpha h_{k} h^{-1}=\left(h h_{1}\right) \alpha\left(h h_{1}\right)^{-1}\left(h h_{2}\right) \alpha\left(h h_{2}\right)^{-1} \ldots$ $\left(h h_{k}\right) \alpha\left(h h_{k}\right)^{-1} \in\langle\alpha: G\rangle$. Therefore $\langle\beta: G\rangle \subseteq\langle\alpha: G\rangle$. 
Lemma 1. Let $G_{1} \leqq G_{2} \leqq S_{n}$ and $\left[G_{2}: G_{1}\right]=2$. Let $\alpha \in T_{n}-S_{n}$. Then $\left\langle\alpha: G_{1}\right\rangle=\left\langle\alpha: G_{2}\right\rangle$ if and only if there is an $h \in G_{2}-G_{1}$ such that $h \alpha h^{-1} \in\left\langle\alpha: G_{1}\right\rangle$.

Proof. If $\left\langle\alpha: G_{1}\right\rangle=\left\langle\alpha: G_{2}\right\rangle$ then for any $h \in G_{2}, h \alpha h^{-1} \in\left\langle\alpha: G_{1}\right\rangle$. To show the converse assume that $h \in G_{2}-G_{1}$ is such that $\beta=h \alpha h^{-1} \in\left\langle\alpha: G_{1}\right\rangle$. Let $p \in G_{2}-G_{1}$. It suffices to show that $p \alpha p^{-1} \in\left\langle\alpha: G_{1}\right\rangle$. Since $h, p \in G_{2}-G_{1}$ and $\left[G_{2}: G_{1}\right]=2$, we have $G_{1} h=G_{1} p$, so there exists $q \in G_{1}$, with $q=p h^{-1}$. Therefore $p \alpha p^{-1}=q h \alpha(q h)^{-1}=$ $q h \alpha h^{-1} q^{-1}=q \beta q^{-1} \in\left\langle\beta: G_{1}\right\rangle \subseteq\left\langle\alpha: G_{1}\right\rangle$, as required.

The following is used to show that if $\alpha \in T_{n}-S_{n}$ then $\left\langle\alpha:\right.$ Alt $\left._{n}\right\rangle=\left\langle\alpha: S_{n}\right\rangle$.

Corollary 2. $\left\langle\alpha: \mathrm{Alt}_{n}\right\rangle=\left\langle\alpha: S_{n}\right\rangle$ if and only if there exists an odd permutation $h$ of $X_{n}$ such that $h \alpha h^{-1} \in\left\langle\alpha: \mathrm{Alt}_{n}\right\rangle$.

Recall that a subgroup $G$ of $S_{n}$ is said to be $k$-transitive if for any two $k$-subsets $A$ and $B$ of $X_{n}$ and any bijection $t$ from $A$ onto $B$, there exists $h \in G$ such that $h(a)=t(a)$ for every $a \in A$. We say that a subgroup $G$ of $S_{n}$ is $k$-block-transitive if for any two $k$-subsets $A$ and $B$ of $X_{n}$ there exists $h \in G$ such that $h(A)=B$. Thus any $k$-transitive semigroup is at least $k$-block-transitive. For example, Alt $_{n}$ is $(n-2)$-transitive $[4,10.4 .6]$, and for all $1 \leqq k \leqq n-1, \mathrm{Alt}_{n}$ is $k$-block transitive.

Given a transformation $\alpha$ of $X_{n}$ denote by $\pi(\alpha)$ the partition of $X_{n}$ determined by $\alpha$ such that $a$ and $b$ are in the same class of $\pi(\alpha)$ if and only if $\alpha(a)=\alpha(b)$. Let im $\alpha=\alpha\left(X_{n}\right)$ be the image of $\alpha$. Note that if $h \in S_{n}$ then $\pi\left(h \alpha h^{-1}\right)=h(\pi(\alpha))=\{h(A): A \in \pi(\alpha)\}$, and $\operatorname{im}\left(h \alpha h^{-1}\right)=h(\operatorname{im} \alpha)$.

Lemma 3. Let $G \leqq S_{n}$ be a k-block transitive group. Then for any $\alpha \in T_{n}-S_{n}$ with $|\operatorname{im} \alpha|=k,\langle\alpha: G\rangle$ contains an idempotent $\beta$ with $\pi(\beta)=\pi(\alpha)$.

Proof. Let $\alpha_{1}(=\alpha), \alpha_{2}, \alpha_{3}, \ldots$ be conjugates of $\alpha$ by elements of $G$ such that im $\alpha_{i}$ is a transversal of $\pi\left(\alpha_{i+1}\right)$ ( $k$-block transitivity of $G$ insures their existence). Consider all the products of the form $\alpha_{1}, \alpha_{2} \alpha_{1}, \alpha_{3} \alpha_{2} \alpha_{1}, \ldots$ Since $\langle\alpha: G\rangle$ is finite there exist integers $m<j$ such that $\alpha_{j} \alpha_{j-1} \ldots \alpha_{m+1} \alpha_{m} \ldots \alpha_{1}=\alpha_{m} \ldots \alpha_{1}$. Let $\delta=\alpha_{j} \ldots \alpha_{m+1}$ and $\gamma=\alpha_{m} \ldots \alpha_{1}$. Then $\delta \gamma=\gamma$ so $\operatorname{im} \delta \supseteq \operatorname{im} \gamma$, and since $|\operatorname{im} \delta|=|\operatorname{im} \alpha|=|\operatorname{im} \gamma|$ we have that $\operatorname{im} \delta=\operatorname{im} \gamma$. Thus $\delta$ is the identity on its image, and so $\delta$ is an idempotent having $\operatorname{im} \delta=\operatorname{im} \alpha_{j}$ and $\pi(\delta)=\pi\left(\alpha_{m+1}\right)$. Let $h \in G$ be such that $\alpha_{m+1}=h \alpha h^{-1}$, then $\beta=h^{-1} \delta h$ is the required idempotent. Indeed $\beta^{2}=h^{-1} \delta h h^{-1} \delta h=h^{-1} \delta^{2} h=h^{-1} \delta h=\beta$ and $\pi(\beta)=\pi\left(h^{-1} \delta h\right)=$ $h^{-1}(\pi(\delta))=h^{-1}\left(\pi\left(\alpha_{m+1}\right)\right)=h^{-1}\left(\pi\left(h \alpha h^{-1}\right)=h^{-1}(\pi(h(\alpha)))=\pi(\alpha)\right.$.

Since $\mathrm{Alt}_{n}$ is $k$-block-transitive for any $1 \leqq k \leqq n-1$ we have the following.

Corollary 4. $\left\langle\alpha: \mathrm{Alt}_{n}\right\rangle$ contains an idempotent $\beta$ with $\pi(\beta)=\pi(\alpha)$.

We say that $\alpha$ has a partition of type $1^{k_{1}} 2^{k_{2}} \ldots r^{k_{r}}$ if $\pi(\alpha)$ has $k_{i}$ classes of size $i$, 
$i=1, \ldots, r[1]$. Note that $\sum_{i=1}^{r} i k_{i}=n$ and we do not exclude the possibility that $k_{i}=0$ for some $i$.

Lemma 5. Let $\alpha \in T_{n}-S_{n}$ be an idempotent. There exists an $h \in S_{n}-\mathrm{Alt}_{n}$ such that $h \alpha h^{-1} \in\left\langle\alpha: \mathrm{Alt}_{n}\right\rangle, n \geqq 3$.

Proof. Assume that there exist $x, y \in \operatorname{im} \alpha$ such that $\alpha^{-1}(x)=\{x\}$ and $\alpha^{-1}(y)=\{y\}$. Then for the transposition $h=(x, y)$ we have $h \alpha h^{-1}=\alpha \in\left\langle\alpha\right.$ : Alt $\left.{ }_{n}\right\rangle$. Now suppose $\pi(\alpha)$ contains a class $A$ having $|A| \geqq 3$. Let $a, b \in A-\operatorname{im} \alpha$. Then for $h=(a, b)$ we have $h \alpha h^{-1}=\alpha \in\left\langle\alpha: \mathrm{Alt}_{n}\right\rangle$.

If none of the above holds then $\alpha$ has a partition of type $1^{0} 2^{k}=2^{k}(k=n / 2, n$ is even) or $1^{1} 2^{k}\left(k=(n-1) / 2, n\right.$ is odd). Let $\alpha_{1}, \alpha_{2}$ be idempotents in $T_{2 k}$ and $T_{2 k+1}$ respectively, $\alpha_{1}=[1,1,3,3, \ldots, 2 k-1,2 k-1]$ and $\alpha_{2}=[1,1,3,3, \ldots, 2 k-1,2 k-1,2 k+1]$ (we write $\left[a_{1}, a_{2}, \ldots, a_{1}\right]$ for a transformation mapping $i$ to $\alpha_{i}$. We may assume without loss of generality that $\alpha$ equals to either $\alpha_{1}$ or $\alpha_{2}$. It is easy to verify that for $h=(12)$ and $n \geqq 5$ we have

$$
h \alpha_{i} h^{-1}=(12)(35) \alpha_{i}(35)(12) \alpha_{i} \in\left\langle\alpha: \mathrm{Alt}_{n}\right\rangle
$$

If $n=4$, then $\alpha_{1}=[1,1,3,3]$, and for $h=(12)$,

$$
h \alpha_{i} h^{-1}=\left((132) \alpha_{1}(123)\right)\left((134) \alpha_{1}(143)\right) \alpha_{1} \in\left\langle\alpha_{1}: \mathrm{Alt}_{4}\right\rangle .
$$

If $n=3, \alpha_{2}=[1,1,3]$, and for $h=(12)$,

$$
h \alpha_{2} h^{-1}=\left((132) \alpha_{2}(123)\right)\left((123) \alpha_{2}(132) \alpha_{2}\right)^{2} \in\left\langle\alpha_{2}: \text { Alt }_{n}\right\rangle .
$$

Proposition 6. Let $\alpha \in T_{n}, n \geqq 3$. Then $\left\langle\alpha: S_{n}\right\rangle$.

Proof. Observe that we only need to show that $\left\langle\alpha: S_{n}\right) \subseteq\left\langle\alpha: \mathrm{Alt}_{n}\right\rangle$. If $\alpha \in \mathrm{Alt}_{n}$ then $\left\langle\alpha: S_{n}\right\rangle \leqq \mathrm{Alt}_{n} \unlhd S_{n}$. Also $\left\langle\alpha: \mathrm{Alt}_{n}\right\rangle \unlhd \mathrm{Alt}_{n}$, and since $\mathrm{Alt}_{n}$ is simple for $n \neq 4[4,10.8 .7]$ we have that $\left\langle\alpha:\right.$ Alt $\left._{n}\right\rangle=$ Alt $_{n}$ if $\alpha \neq(1)$ and $\left\langle(1):\right.$ Alt $\left._{n}\right\rangle=\{(1)\}$ (provided $n \neq 4$ ). If $n=4, \alpha \neq(1)$ and $\left\langle\alpha:\right.$ Alt $\left._{4}\right\rangle \neq \mathrm{Alt}_{4}$ then $\left\langle\alpha:\right.$ Alt $\left._{4}\right\rangle=V$, the 4-group, and $\alpha \in V$. Since V $\unlhd S_{4},\left\langle\alpha: S_{4}\right) \leqq V$ also, and therefore $\left\langle\alpha: S_{4}\right\rangle \subseteq\left\langle\alpha:\right.$ Alt $\left._{4}\right\rangle$, as required.

If $\alpha$ is an odd permutation then for any $q \in S_{n}-\mathrm{Alt}_{n}, q=\alpha\left(\alpha^{-1} q\right), \alpha^{-1} q \in \mathrm{Alt}_{n}$, and $q \alpha q^{-1}=\alpha\left(\alpha^{-1} q\right) \alpha\left(\alpha^{-1} q\right)^{-1} \alpha^{-1} \in\langle\alpha:$ Alt $n)$, so $\left\langle\alpha: S_{n}\right\rangle \subseteq\langle\alpha:$ Alt $n\rangle$ again.

Now let $\alpha \in T_{n}-S_{n}$. By Corollary 4, $\left\langle\alpha\right.$ : Alt $\left.{ }_{n}\right\rangle$ contains an idempotent $\beta$ with $\pi(\beta)=\pi(\alpha)$. By Lemma 5 and Corollary 2, $\left\langle\alpha:\right.$ Alt $\left._{n}\right\rangle \supseteq\left\langle\beta:\right.$ Alt $\left._{n}\right\rangle=\left\langle\beta: S_{n}\right\rangle=\left\langle\alpha: S_{n}\right\rangle$ (for a transformation $\gamma$ the semigroup $\left\langle\gamma: S_{n}\right\rangle$ comprises all $\delta \in T_{n}$ having $\pi(\delta) \supseteq Q$, a partition of the same type as $\pi(\gamma)[2])$.

Corollary 7. There is no $S \subseteq T_{n}$ such that $G_{S}=\mathrm{Alt}_{n}$.

Proof. Suppose $\mathrm{Alt}_{n} \subseteq G_{S}$. Then by Proposition 6, for any $\alpha \in S, h \in S_{n}$, we have that $h \alpha h^{-1} \in\left\langle\alpha: S_{n}\right\rangle=\left\langle\alpha: A \mid t_{n}\right\rangle \subseteq S$, that is $h \in G_{S}$ and $G_{S}=S_{n}$. 
Now to prove our main Theorem suppose that $G \leqq \operatorname{Inn} S$ such that $G \cong \mathrm{Alt}_{n}$, Let $\bar{G}=\left\{h \in S_{n}: \phi_{h} \in G\right\}$. Then $\bar{G} \leqq G_{S} \leqq S_{n}$, and the order of $\bar{G}$ is at least that of $\mathrm{Alt}_{n}$. Therefore $\bar{G}$ contains $\mathrm{Alt}_{n}$, and by Corollary $7, G_{S}=S_{n}$.

We note that the above result is not necessarily true for semigroups of transformations of infinite sets. For example, let $X=\mathbf{Z}$ be the set of all integers and $\alpha: \mathbf{Z} \rightarrow \mathbf{Z}$ such that $\alpha(a)=2 a$, for all $a \in Z$. Let $S_{\mathbf{Z}}$ be the symmetric group on $\mathbf{Z}$. The alternating subgroup $A t_{\mathrm{Z}}$ of $S_{\mathrm{Z}}$ consists of all the finite even permutations of $\mathbf{Z}$. Then $\left\langle\alpha: S_{\mathrm{Z}}\right\rangle=$ $\left\{\beta: \mathbf{Z} \rightarrow \mathbf{Z} \mid \beta\right.$ is $1-1$ and $\left.|\mathbf{Z}-\operatorname{im} \beta|=\aleph_{0}\right\}$ [3]. In particular $\left\langle\alpha: S_{\mathbf{Z}}\right\rangle$ contains $\beta$ defined by $\beta(a)=2 a-1$ for all $a \in \mathbf{Z}$. Observe that for all $a \in \mathbf{Z}, \alpha(a) \neq \beta(a)$. Since any $h \in \mathrm{Alt}_{\mathbf{Z}}$ moves at most a finite number of points, $\beta \notin\left\langle\alpha\right.$ : Alt $\left._{\mathbf{z}}\right\rangle$.

For a transformation $\alpha$ of $X$ let shift $\alpha=|\{x \in X: \alpha(x) \neq x\}|$. Let $v$ be an infinite cardinal not exceeding $|X|^{+}$, the cardinal successor of $|X|$, and let $\operatorname{Sym}(X, v)$ be the subgroup of all permutations in $S_{X}$ whose shift is less than $v$.

Conjecture 1. If shift $\alpha=u$ then $\langle\alpha: \operatorname{Sym}(X, w)\rangle=\left\langle\alpha: S_{X}\right\rangle$ for all $w \geqq u^{+}$.

2. There is no semigroup $S$ of transformations of $X$ having $G_{S}=\operatorname{Sym}(X,|X|)$.

Observe that permutations $h$ and $p$ in $G_{S}$ give rise to equal automorphisms $\phi_{h}$ and $\phi_{p}$ if and only if $h^{-1} p$ is in the centralizer $C(S)$ of $S, C(S)=\left\{\alpha \in T_{n}: \alpha \beta=\beta \alpha\right.$ for all $\left.\beta \in S\right\}$. Thus $G_{S}$ is isomorphic to the group $\operatorname{Inn} S$ of the inner automorphisms of $S$ if and only if $C(S) \cap G_{S}$ consists of the identity permutation. The results of this paper in conjunction with the above observations give rise to the following.

Problem 1. Characterize these subgroups $G$ of $S_{n}$ having $G=G_{S}$ for some subsemigroup $S$ of $S_{n}$.

2. Given that $G=G_{T}$ for some $T \subseteq T_{n}$ characterize all $S \subseteq T_{n}$ such that $G_{S}=G$.

3. Characterize these subsemigroups $S$ of $T_{n}$ having $\left|C(S) \cap G_{S}\right|=1$.

\section{REFERENCES}

1. I. Anderson, Combinatorics of Finite Sets, Oxford Science Publications, 1989.

2. I. Levi and R. McFadden, $S_{n}$-normal semigroups, Proc. Edinburgh Math. Soc. 37 (1994), 471-476.

3. I. Levı, Normal semigroups of one-to-one transformations, Proc. Edinburgh Math. Soc. 34 (1991), 65-76.

4. W. R. Scott, Group Theory (Dover Publications, 1987).

5. R. P. Sullivan, Automorphisms of transformation semigroups, J. Austral. Math. Soc. Ser. A 20 (1975), 77-84.

6. J. S. V. Symons, Normal transformation semigroups, J. Austral. Math. Soc. Ser. A 22 (1976), 385-390.

Mathematics Department

UNIVERSITY OF LOUISVILLE

LOUISVILLE, KY 40292

U.S.A. 